\title{
Identification of Siglec-9 as the receptor for MUC16 on human NK cells, B cells, and monocytes
}

\author{
Jennifer A Belisle1, Sachi Horibata', Gubbels AA Jennifer'1, Sarah Petrie1', Arvinder Kapur'1, Sabine André2, Hans- \\ Joachim Gabius², Claudine Rancourt33, Joseph Connor ${ }^{1}$, James C Paulson ${ }^{4}$ and Manish S Patankar*1
}

\begin{abstract}
Background: MUC16 is a cell surface mucin expressed at high levels by epithelial ovarian tumors. Following proteolytic cleavage, cell surface MUC16 (csMUC16) is shed in the extracellular milieu and is detected in the serum of cancer patients as the tumor marker CA125. csMUC16 acts as an adhesion molecule and facilitates peritoneal metastasis of ovarian tumors. Both sMUC16 and csMUC16 also protect cancer cells from cytotoxic responses of natural killer (NK) cells. In a previous study we demonstrated that sMUC16 binds to specific subset of NK cells. Here, we identify the csMUC16/sMUC16 binding partner expressed on immune cells.

Results: Analysis of immune cells from the peripheral blood and peritoneal fluid of ovarian cancer patients indicates that in addition to NK cells, sMUC16 also binds to B cells and monocytes isolated from the peripheral blood and peritoneal fluid. I-type lectin, Siglec-9, is identified as the sMUC16 receptor on these immune cells. Siglec-9 is expressed on approximately $30-40 \%$ of CD16 pos/CD56 dim NK cells, $20-30 \%$ of B cells and $>95 \%$ of monocytes. SMUC16 binds to the majority of the Siglec-9pos NK cells, B cells and monocytes. sMUC16 is released from the immune cells following neuraminidase treatment. Siglec-9 transfected Jurkat cells and monocytes isolated from healthy donors bind to ovarian tumor cells via Siglec-9-csMUC16 interaction.

Conclusions: Recent studies indicate that csMUC16 can act as an anti-adhesive agent that blocks tumor-immune cell interactions. Our results demonstrate that similar to other mucins, csMUC16 can also facilitate cell adhesion by interacting with a suitable binding partner such as mesothelin or Siglec-9. Siglec-9 is an inhibitory receptor that attenuates T cell and NK cell function. sMUC16/csMUC16-Siglec-9 binding likely mediates inhibition of anti-tumor immune responses.
\end{abstract}

\section{Introduction}

MUC16 is a membrane spanning mucin that is expressed on ovarian, endometrial, tracheal, and ocular surface epithelial cells [1-4]. This mucin is initially expressed on the surface (cell surface MUC16, csMUC16) and then shed (shed MUC16, sMUC16) in the extracellular milieu following proteolytic cleavage [5-7]. csMUC16 carries a $\sim 12,000$ amino acid N-terminal region, a Variable Number of Tandem Repeat (VNTR) domain composed of 60 tandem repeats of 156 amino acids, and a 256 amino acid cytoplasmic tail [7-9]. The mucin is heavily glycosylated with both $\mathrm{O}$ - and $\mathrm{N}$-linked oligosaccharides [10]. Because of all of these structural features, the average molecular

\footnotetext{
* Correspondence: patankar@wisc.edu

1 Department of Obstetrics and Gynecology, University of Wisconsin-Madison, Madison, Wl; USA

Full list of author information is available at the end of the article
}

weight of csMUC16 and sMUC16 is between 3-5 million $\mathrm{Da}[7,9,10]$.

csMUC16 is overexpressed by human epithelial ovarian tumor cells [11,12]. sMUC16 is released by ovarian tumors and can be detected in the peritoneal fluid and peripheral blood of cancer patients. A repeating peptide epitope present in the VNTR domain of csMUC16 and sMUC16 has been previously identified as the tumor marker CA125. Elevations, from an individualized nadir serum concentration of CA125, are routinely determined in order to monitor progression of epithelial ovarian cancer in patients undergoing treatment for this disease $[13,14]$.

In addition to serving as a cancer biomarker, MUC16 is also important in promoting the metastasis and growth of ovarian tumors. csMUC16 facilitates tumor cell aggregation and their subsequent binding to the peritoneal

@ 2010 Belisle et al; licensee BioMed Central Ltd. This is an Open Access article distributed under the terms of the Creative Commons B. Wed Central Attribution License (http://creativecommons.org/licenses/by/2.0), which permits unrestricted use, distribution, and reproduction in any medium, provided the original work is properly cited. 
surfaces by serving as a ligand of mesothelin, a glycoprotein expressed on mesothelial cells [15-17]. Both csMUC16 and sMUC16 protect ovarian tumor cells from immune attack and thereby promote tumor growth. sMUC16 is an inhibitor of the cytolytic anti-tumor responses of natural killer (NK) cells [18]. On the other hand, csMUC16 acts as an anti-adhesive molecule and prevents the formation of the immunological synapse between ovarian cancer cells and NK cells [19]. Additional studies are required to carefully delineate the immunoprotective role of sMUC16 and csMUC16 and their contribution to the progression of ovarian tumorigenesis.

Phenotypic analysis of NK cells isolated from the peripheral blood and peritoneal fluid of ovarian cancer demonstrated that sMUC16 derived from the tumors binds strongly to the surface of a select subset of CD16 pos/CD56 dim NK cells [20]. RT-PCR of peripheral blood mononuclear cells (PBMC) from ovarian cancer patients and other in vitro experiments, reported in our previous study, showed that immune cells do not express MUC16 [20]. sMUC16 was detected on the immune cell surface using the murine monoclonal antibodies VK8, and OC125 [20]. Both VK8 and OC125 are highly specific anti-MUC16 antibodies. VK-8 was used in experiments that ultimately led to the cloning of MUC16 [6,21] and the OC125 antibody was used as a reagent to establish the clinical-grade CA125 assay to measure the concentration of this marker in the sera of cancer patients $[22,23]$. A third antibody, 618F, developed by our group [24], also binds specifically to subsets of CD16 pos/CD56 dim NK cells isolated from ovarian cancer patients but not to those obtained from healthy donors (Patankar et al unpublished data). Lack of endogenous MUC16 expression and the binding of highly specific anti-MUC16 antibodies to only fixed subsets of patient derived immune cells suggested the presence of a MUC16 receptor on immune cells. The current study was therefore undertaken to identify the binding partner for MUC16 on immune cells.

Here, we report that sMUC16 selectively binds not only to a subset of CD16 pos/CD56 dim NK cells but also is present on the surface of approximately $20 \%$ of the CD19pos B cells and $>90 \%$ of all CD14pos monocytes. Additional experiments demonstrate that both sMUC16 and csMUC16 bind to the immune cell surface via the I-type lectin Siglec-9. csMUC16-Siglec-9 binding mediates adhesion of immune cells with ovarian tumor cells. Identification of Siglec-9 as the sMUC16/csMUC16 binding partner provides an opportunity to identify the molecular mechanisms that lead to suppression of immune responses by this mucin. Apart from its importance in understanding the biological role of MUC16, the data also suggests that sMUC16 binding to immune cells may serve as a novel strategy for the detection and monitoring of ovarian cancer.

\section{Results}

\section{Potential sMUC16 receptors on CD16 pos/CD56 dim NK cell} surface

Human NK cells are classified into two major phenotypes, CD16 pos/CD56 dim and CD16 neg/CD56 bright. The CD16 pos/CD56 dim NK cells are highly cytotoxic NK cells whereas the CD16 $6^{\text {neg }} / \mathrm{CD} 56^{\text {bright }} \mathrm{NK}$ cells are not efficient in lysing tumor targets $[25,26]$. Because sMUC16 binds only to subsets of CD16 pos/CD56 dim NK cells and also inhibits their cytolytic activity, we postulated that sMUC16 is likely a ligand of an inhibitory receptor on NK cells. Since sMUC16 is heavily glycosylated, we specifically considered carbohydrate binding receptors of NK cells as potential binding partners.

The lectin galectin-1 has been reported as a MUC16 receptor on NK cells $[27,28]$. However, galectin-1 is primarily expressed by CD16 dim/CD56 bright NK cells (Additional file 1) while MUC16 is bound by CD16 pos/CD56 dim NK cells. Therefore, galectin-1 was considered unlikely to be involved in mediating binding of sMUC16 to CD16 CD56 dim NK cells. Another binding partner for sMUC16 is mesothelin [15]. This glycoprotein is however not expressed on NK cells based on microarray analysis [29].

To test if sMUC16 was binding to immune cells via its oligosaccharides, we digested the peripheral blood mononuclear cells (PBMC) from ovarian cancer patients with neuraminidase from Clostridium perfringens. After a limited (15 min) digestion with neuraminidase, the PBMC were monitored by flow cytometry for the amount of sMUC16 still bound to the cell surface. Neuraminidase treatment resulted in a loss of sMUC16 binding on approximately $50 \%$ of the PBMCs as compared to matching controls not treated with neuraminidase (Figure 1).

NK cells are reported to express two members of the sialic acid binding Siglec family, Siglec-7 and Siglec-9 $[30,31]$. Siglec-9 recognizes $\alpha 2-3$-linked sialic acid where as Siglec-7 preferentially binds to glycans terminated with $\alpha 2-8$-linked sialic acid residues [32,33]. Glycomic analysis has revealed extensive expression of $\alpha 2$-3-linked sialic acid residues on MUC16 [10]. Therefore, we focused our attention on Siglec- 9 as a potential candidate immune cell receptor for this mucin.

\section{sMUC16 predominantly binds to Siglec-9pos/CD16pos/ CD56 dim NK cells}

Analysis of NK cells from the peripheral blood (PB) of four healthy donors indicated that Siglec-9 was detectable only on approximately $15-40 \%$ of the CD16 pos/CD56 dim cells (Figure $2 \mathrm{~A}$ ). The $\mathrm{CD} 16^{\mathrm{neg}} / \mathrm{CD} 56^{\text {bright }}$ NK cells were either negative or presented very low levels of Siglec-9 


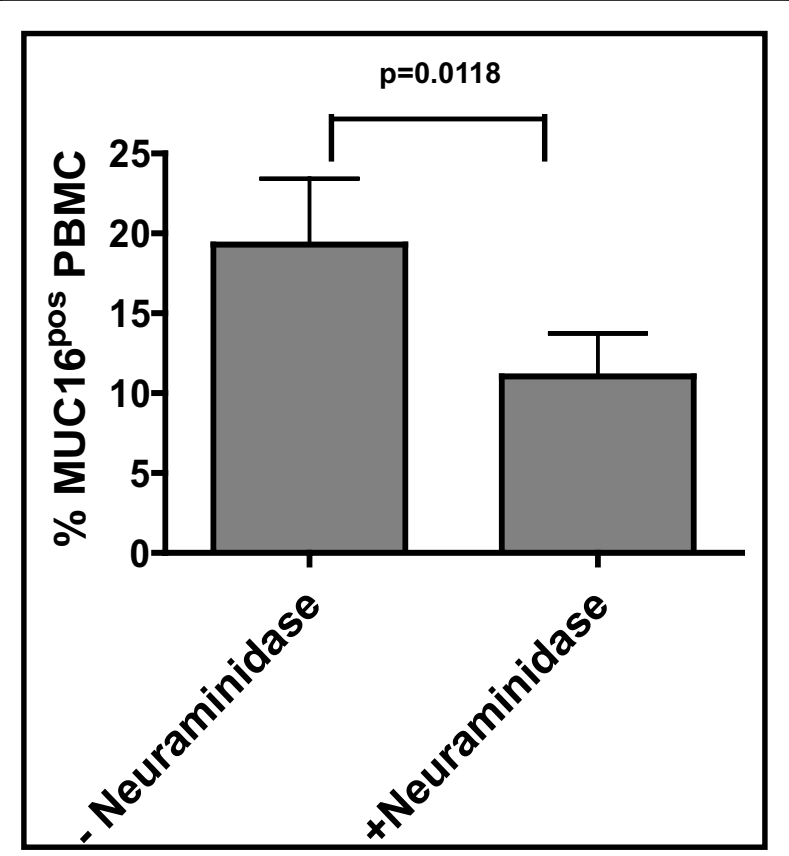

Figure 1 sMUC16 binding to immune cells is reduced following neuraminidase treatment. PBMC from each of six ovarian cancer patients were digested with neuraminidase from Clostridium perfringens in phosphate buffered saline (PBS). Matching control PBMC from each of the six patients were treated with heat inactivated neuraminidase (Clostridium perfringens). sMUC16 binding to the control and enzymatically digested cells was measured by flow cytometry using the antiMUC16 antibody, VK-8. Each bar is the mean measurement for all six donors. Data was analyzed using the Wilcoxon signed rank test.

(Figure 2A). A similar expression pattern for Siglec-9 was also observed on NK cells from $\mathrm{PB}$ and peritoneal fluid (PF) of epithelial ovarian cancer patients (Figure 2A).

As mentioned previously, sMUC16 is detected only on $30-40 \%$ of the CD16pos/CD56 dim NK cells isolated from the $\mathrm{PB}$ and $\mathrm{PF}$ of ovarian cancer patients, the same subset of NK cells that also are likely to express Siglec-9. We therefore performed multi-color flow cytometry experiments to determine if sMUC16 was selectively binding to the Siglec-9pos/CD16pos/CD56dim NK cell subset. While Siglec-9 was detected on the CD16 ${ }^{\text {pos }} / C D 56^{\text {dim }}$ NK cells isolated from the $\mathrm{PB}$ of healthy donors, the level of sMUC16 on these cells was either undetectable or very low (Figure 2B, C). To the contrary, between $74-82 \%$ of the Siglec-9pos/CD16 ${ }^{\text {pos } / C D 56 ~}{ }^{\text {dim }}$ NK cells isolated from the PB and PF of five ovarian cancer patients were also positive (double positive) for sMUC16 (Figure 2C). The differences between the percentage of patient $\mathrm{PB}$ - and PF-derived CD16 pos/CD56 dim NK cells that were positive for Siglec-9 and sMUC16 were statistically not significant (Figure 2C).

\section{Siglec-9 expression on B cells and monocytes}

The PB and PF derived mononuclear cells (PBMC and PFMC) contain B cells, $\mathrm{T}$ cells, and monocytes in addi- tion to the NK cells. Only a very minor subset (less than 2\%) of the PBMC and PFMC derived CD3pos $\mathrm{T}$ cells expressed Siglec-9 (data not shown). Furthermore, only low levels of Siglec-9 was observed on these T cells. We generally did not observe sMUC16 binding to the small percentage of these Siglec-9pos T cells. However, because of the low number of events of Siglec-9pos $\mathrm{T}$ cells and low levels of expression of this receptor, we were unable to obtain statistically relevant information on sMUC16 binding to $\mathrm{T}$ cells of all of the healthy donors and ovarian cancer patients recruited in this study. Hence, T cells were not further analyzed for sMUC16 binding and Siglec-9 expression.

Approximately 20\% of CD19pos B cells and greater than $90 \%$ of the CD33pos monocytes were Siglec-9pos (Figure 3). The expression level of Siglec-9 on B cells and NK cells was comparable. However, the entire population of the CD33pos monocytes expressed several fold higher levels of Siglec- 9 as compared to the B cells and NK cells (Figure $3)$.

\section{sMUC16 is present on Siglec-9pos B cells and monocytes} If sMUC16 was binding to NK cells via Siglec-9, this mucin should also be present on the Siglec-9pos B cells and monocytes. This was indeed the case since $65-70 \%$ of the total sMUC16 ${ }^{\text {pos B }}$ cells were also Siglec-9pos (Figure $4 \mathrm{~A})$. In the case of monocytes the correlation between sMUC16 binding and Siglec-9 expression was even more striking. Greater than $95 \%$ of the sMUC16 ${ }^{\text {pos }}$ monocytes were Siglec-9pos and vice versa (Figure 4B and 4C). Overall, the mean fluorescent intensity for sMUC16 binding to the B cells, NK cells, and monocytes matched the expression levels of Siglec-9 on these immune cell types (data not shown).

\section{sMUC16 binds to Siglec-9}

In Western blot analysis sMUC16 purified from OVCAR3 cells was detected by the Siglec-9-human Fc chimera (Figure 5A). Fetuin, an $\alpha 2,3$-sialic acid expressing glycoprotein, blocked the binding of Siglec-9 human Fc chimera to sMUC16 (Figure 5B). Asialofetuin under identical conditions did not abrogate the binding of Siglec-9-human Fc to sMUC16 (Figure 5B).

To further demonstrate that Siglec-9-human Fc was recognizing the sialic acids of sMUC16 the mucin was digested with neuraminidase. Efficient desialylation of sMUC16 was demonstrated by the increased migration of asialo-sMUC16 detected by Western blot analysis using the anti-MUC16 antibody VK-8 (Figure 5C). Enzymatically desialylated sMUC16 was not recognized by the Siglec-9-human Fc chimera (Figure 5C).

Specificity of sMUC16 binding to Siglec-9 was determined using Jurkat cells transfected with cDNA coding for this I-type lectin [34]. The Siglec-9 expressing Jurkat cells were incubated with either sMUC16 $(50,000 \mathrm{U}$ of 


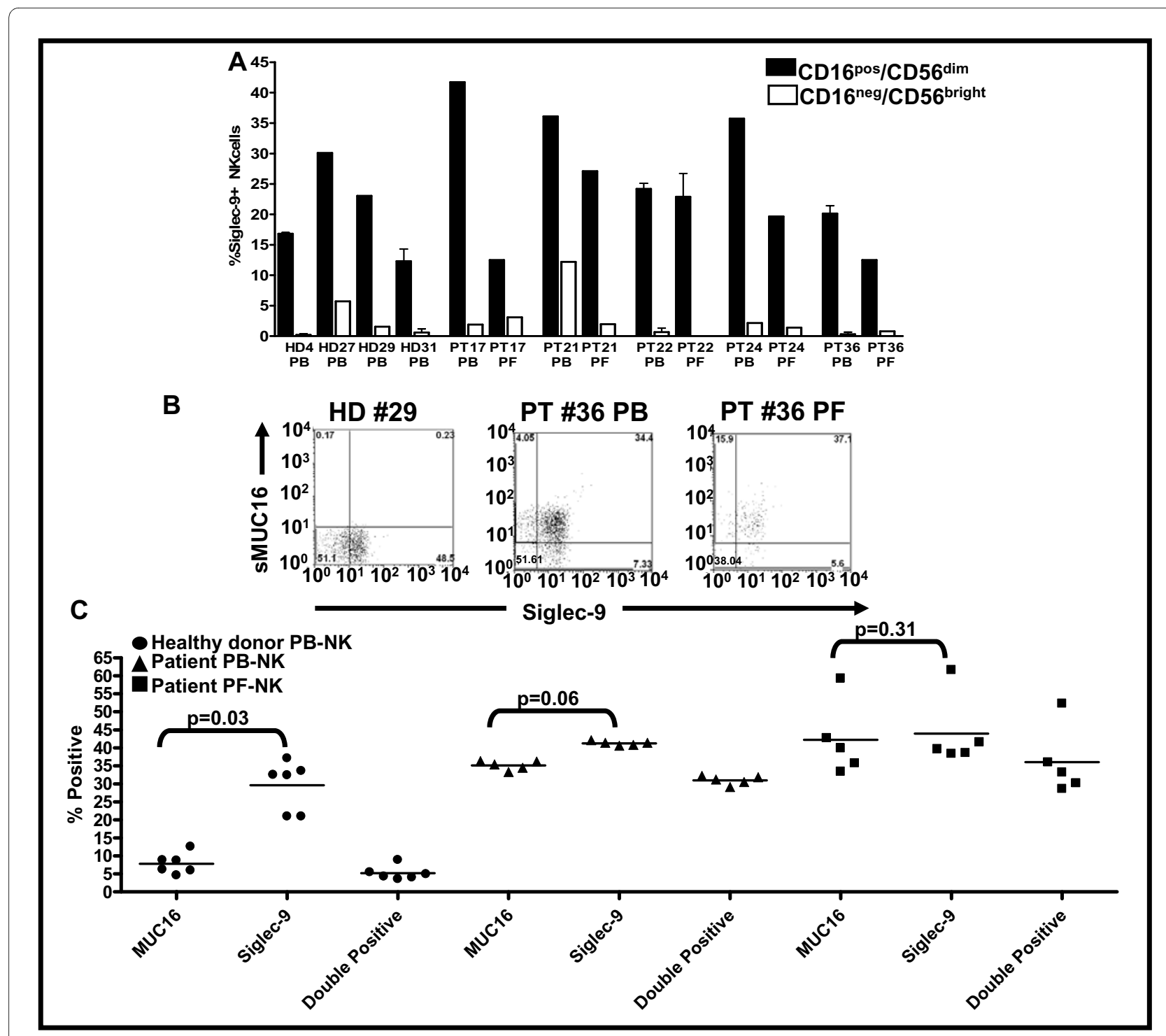

Figure 2 sMUC16 predominantly binds to Siglec-9pos/CD16 pos/CD56 dim NK cells. Peripheral blood mononuclear cells (PBMC) from healthy donors (HD) and patients (PT) and peritoneal fluid mononuclear cells (PFMC) of PT were labeled with VK8 followed by a fluorophore conjugated secondary reporter antibody. The cells were subsequently labeled with fluorescently tagged anti-CD3,-CD16, -CD56, -Siglec-9, and -NKp46 antibodies as described earlier [20]. NK cells identified as CD3neg/NKp46pos cells were classified into CD16pos/CD56 dim and CD16 neg/CD56bright cells by flow cytometry. $\boldsymbol{A}$, Siglec-9 expression on the two NK cell subpopulations, CD16 pos/CD56 dim and CD16 neg/CD56 bright, was analyzed. Each bar represents a mean and standard deviation of three independent experiments. B and C, sMUC16 binding and Siglec-9 expression on CD16pos/CD56dim NK isolated from PBMC of healthy donors and PBMC and PFMC of cancer patients was determined. B, shows dot plots of representative healthy donor (HD) peripheral blood NK and patient (PT) NK cells from peripheral blood (PB) and peritoneal fluid (PF). Majority of events in the lower left quadrant are located on the axes and therefore not visible in the dot plots. $C$, Each value is mean of PBMC from 6 healthy donor and PBMC and PFMC from five cancer patients showing percent of the cells positive for Siglec-9 and sMUC16 and those double positive for both Siglec-9 and sMUC16 are shown.

CA125/ml) purified from the conditioned media of OVCAR-3 cells or with peritoneal fluid from two ovarian cancer patients. After culture for $24 \mathrm{~h}$ (Figure 6A), sMUC16 was detected on Siglec-9 expressing Jurkat cells. Matched Jurkat cells that expressed Siglec-7 instead of Siglec-9 did not show any binding of sMUC16 under identical conditions (Figure 6A).
To further demonstrate that sMUC16 was binding to Siglec-9 expressing Jurkat cells via its sialic acid residues, purified mucin was desialylated with neuraminidase from Clostridium perfringens. The limited availability of purified sMUC16 compelled us to conduct neuraminidase treatment in cell culture compatible buffer $(\mathrm{pH}$ 7.4). The neuraminidase has an optimum $\mathrm{pH}$ of 5 [35]. Hence, 

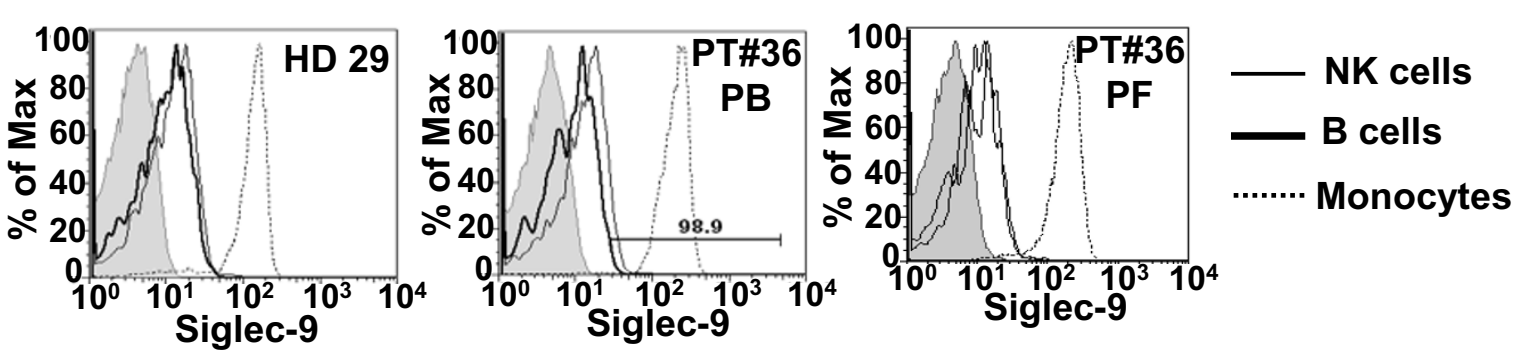

Figure 3 Expression of Siglec-9 on NK cells, B cells and monocytes. PBMC from healthy donors (HD) and PBMC and PFMC from patients (PT) were labeled with fluorophore conjugated anti-CD3, -CD19, -CD16, -CD33, -CD45, -CD56, and -Siglec-9 antibodies. Cells were analyzed by flow cytometry. Live and single events were gated and expression of Siglec-9 on CD16 pos/CD56 dim (NK) cells, CD19pos B cells, and CD33pos monocytes was determined. Data shown for HD \#29 and PT\#36 is representative of that obtained for four healthy donors and five ovarian cancer patients, respectively.

digestion with this neuraminidase at $\mathrm{pH} 7.4$ yielded only partial desialylation of sMUC16- as confirmed by western blotting experiments with VK-8 and biotinylated Mackia amurensis agglutinin (data not shown). The partially desialylated sMUC16 exhibited a $20-50 \%$ reduction in the binding to Siglec-9 expressing Jurkat cells (Figure 6B).

\section{Siglec-9 promotes immune cell-tumor cell adhesion via csMUC16}

MUC1 binding to Siglec-4a (myelin associated glycoprotein) contributes to perineural adhesion of pancreatic cancer cells [36]. We therefore investigated if Siglec-9 could also promote immune cell-tumor cell adhesion via csMUC16 expressed on ovarian cancer cells.

To demonstrate the ability of Siglec-9 to act as a cell binding receptor, we utilized MUC16 knock down OVCAR-3 subline csMUC16 ${ }^{\text {negOVC }}$ and MUC16

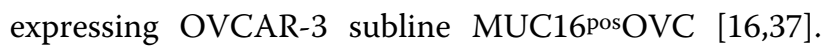
The csMUC16 ${ }^{\text {neg }} \mathrm{OVC}$ cells express an endoplasmic reticulum localized scFv fragment of the VK8 antibody that prevents expression of csMUC16 and sMUC16. The

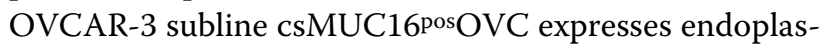
mic reticulum localized $\mathrm{scFv}$ fragment of an irrelevant mouse antibody that does not interfere with csMUC16 and sMUC16 expression.

Using two separate cell adhesion assays it was determined that Siglec-9 expressing Jurkat cells formed approximately 1.5-2-fold more cell conjugates with csMUC16 ${ }^{\text {pos }} \mathrm{OVC}$ cells as compared to csMUC16 ${ }^{\text {neg }} \mathrm{OVC}$ cells. Siglec-7 expressing Jurkat cells, most likely via Siglec-7-independent mechanisms, did indeed form conjugates with both csMUC16 negOVC and csMUC16 posOVC cells (Figure 7A, B). However, significantly more conjugate formation was observed between Siglec-9 expressing Jurkat cells and csMUC16posOVC cells than between Siglec-7 expressing Jurkat and csMUC16 pos OVC cells (Figure 7A, B).
Cell binding assays were also performed with monocytes isolated from the PB of healthy donors. Similar to results obtained with the Siglec-9 and Siglec-7 expressing Jurkat cells, basal level of binding of monocytes was


independent experiments conducted with monocytes isolated from three healthy donors, increased binding of the monocytes to the csMUC16 ${ }^{\text {pos-OVC }}$ as compared to the csMUC16 ${ }^{\text {neg-OVC }}$ was consistently observed. The increased binding of monocytes to the csMUC16 1 pos-OVC $^{-O}$ was significantly inhibited by an anti-Siglec- 9 blocking antibody (Figure 8) [38].

\section{Discussion}

Mucins are bulky, heavily glycosylated molecules that are known to function in diverse biological scenarios $[39,40]$. One of the intriguing properties of mucins is their ability to act as both adhesive as well as anti-adhesive molecules. The bulky nature of the mucins coupled with the presence of negatively charged oligosaccharides contributes to their anti-adhesive properties. On the other hand, specific oligosaccharide and peptide sequences of mucins may serve as ligands for cell adhesion receptors such as the selectins and integrins thereby allowing mucins to act as pro-adhesion molecules.

Similar to other mucins, MUC16 also exhibits both anti- and pro-adhesive properties. By serving as a ligand of mesothelin, MUC16 mediates binding between ovarian cancer cells and the mesothelium $[15,16]$. The antiadhesive property of MUC16 has been clearly demonstrated in the human endometrial tissues [3]. Our recent work [19] shows that the anti-adhesive property of MUC16 allows the tumor cells to circumvent immune synapse formation with cytotoxic NK cells.

In the current study, we have now identified a new mechanism by which csMUC16 may increase the contacts between ovarian cancer cells and immune cells. 
Belisle et al. Molecular Cancer 2010, 9:118

Page 6 of 14

http://www.molecular-cancer.com/content/9/1/118

A
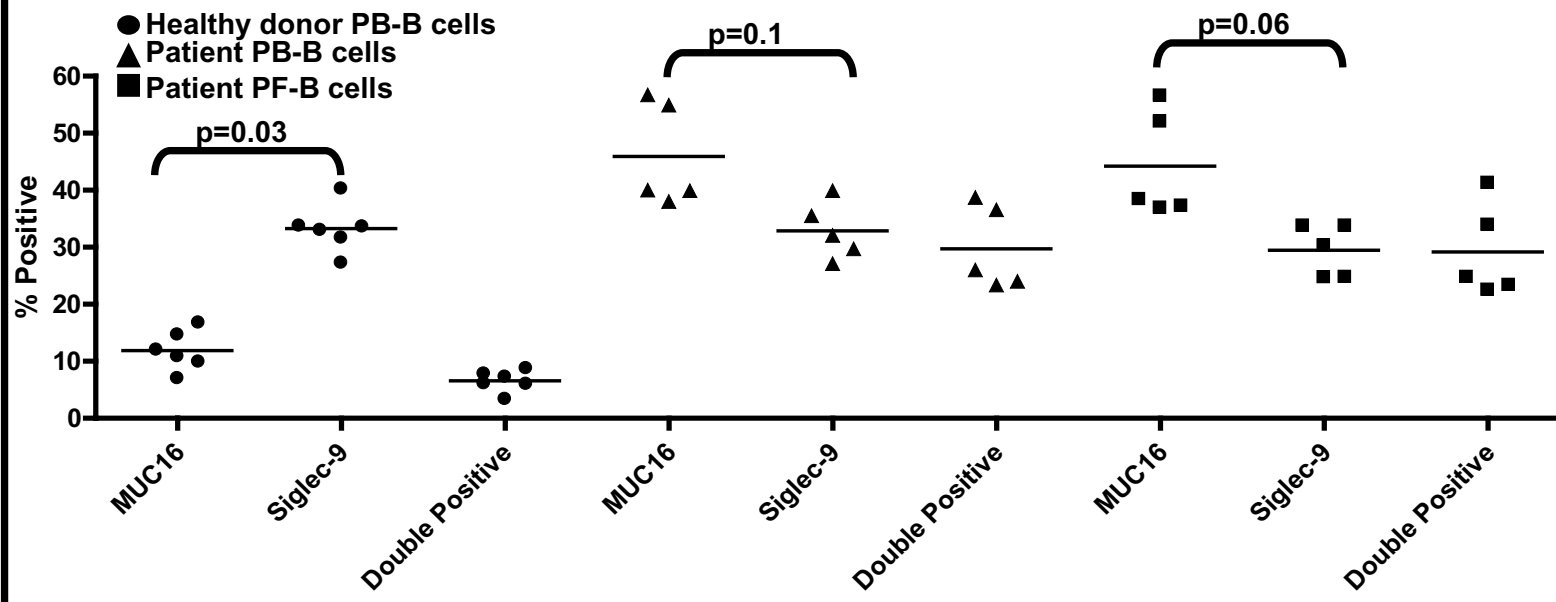

B
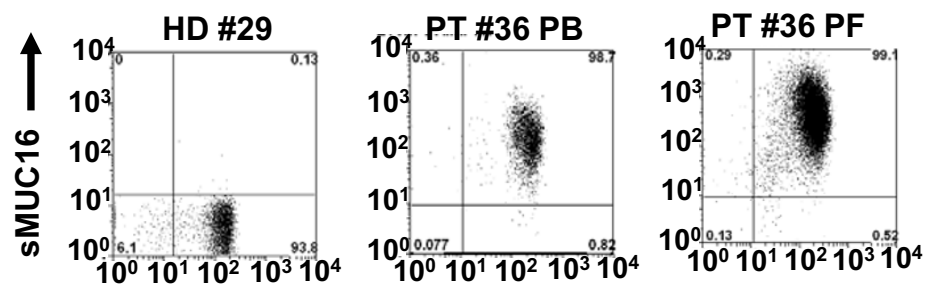

Siglec-9

C

- Healthy donor PB-monocytes

Patient PB-monocytes

Patient PF-monocytes

$\mathrm{p}=0.03$
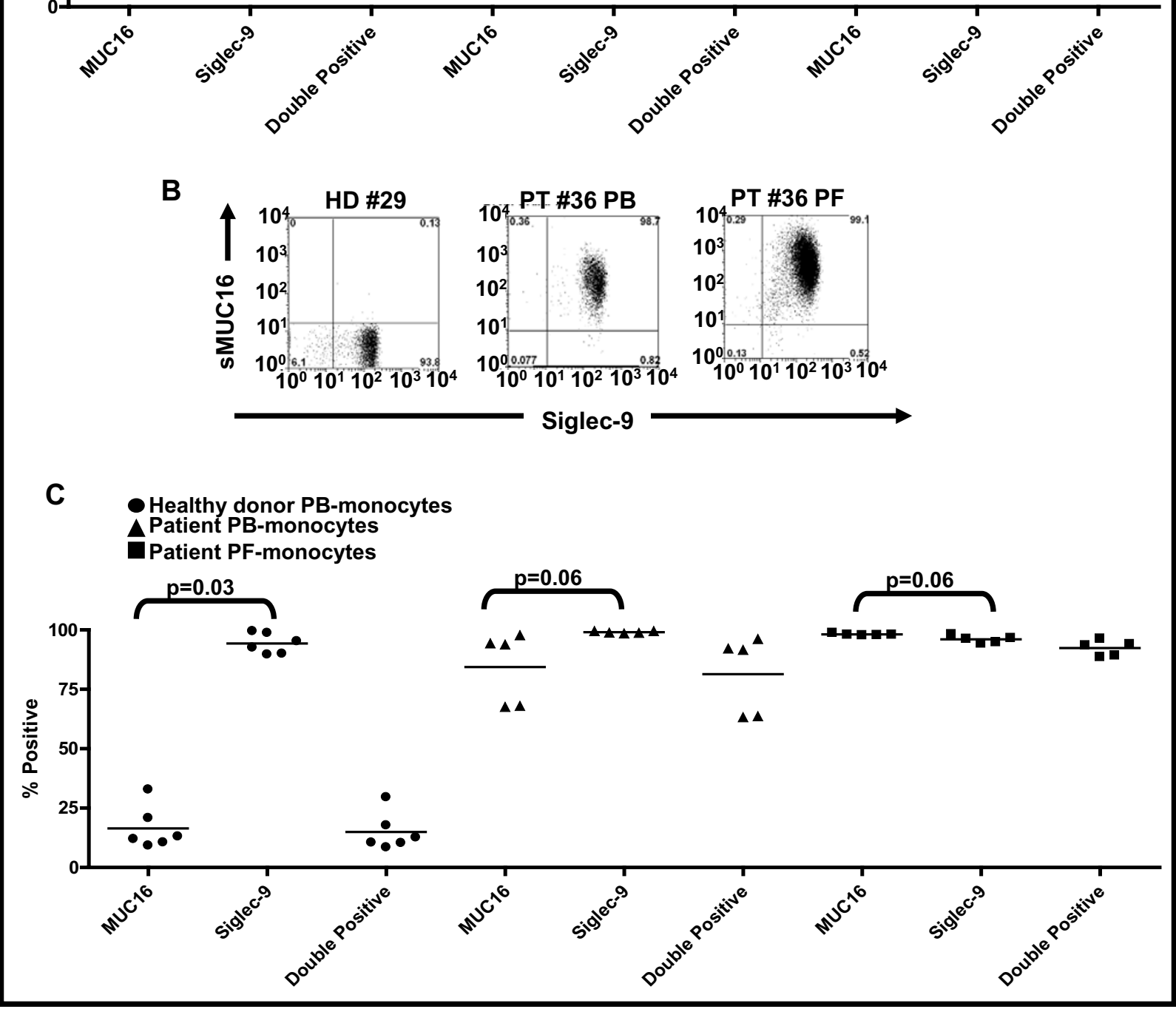

Figure 4 sMUC16 predominantly binds to Siglec-9pos B cells and monocytes. B cells (CD19pos) and monocytes (CD33pos) present in the PBMC of healthy donors (HD) and PBMC and PFMC of ovarian cancer patients (PT) were identified by flow cytometry. $\boldsymbol{A}$, Siglec-9 expression and sMUC16 binding on B cells was determined and double positive events were gated. Data shown is mean of percent positive events for six HD and five PT PBMC samples. $\boldsymbol{B}$, High level of expression of Siglec-9 and a correspondingly high level of sMUC16 binding was observed on monocytes. Data shown is for HD \#29 and PT \#36 and is representative of the cumulative data, $\mathbf{C}$, obtained for six HD and five PT. 


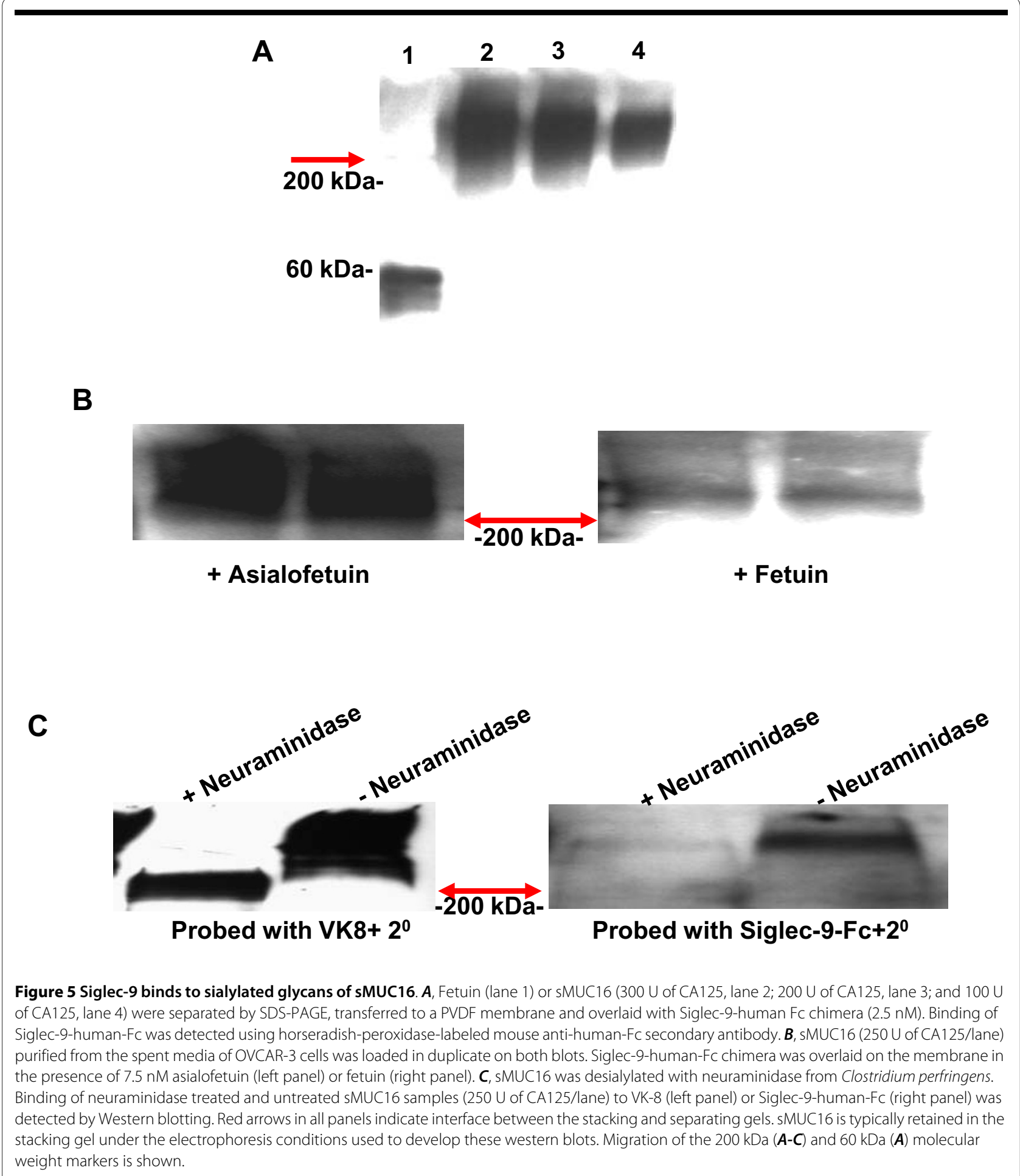

Here, we have identified Siglec-9 as the immune cell receptor for sMUC16 and csMUC16. The binding of csMUC16 to Siglec-9 allows immune cells to form direct contact with the ovarian cancer cells. Thus, while on the one hand, csMUC16 blocks immune synapse formation, the results of the current study indicate that the mucin may allow increased immune cell binding to ovarian cancer cells. These seemingly contradictory observations can, however, be reconciled.

Siglec-9 is an inhibitory receptor that signals via its proximal Immunoreceptor Tyrosine-based Inhibition Motif (ITIM). Ligand binding results in the phosphoryla- 
A
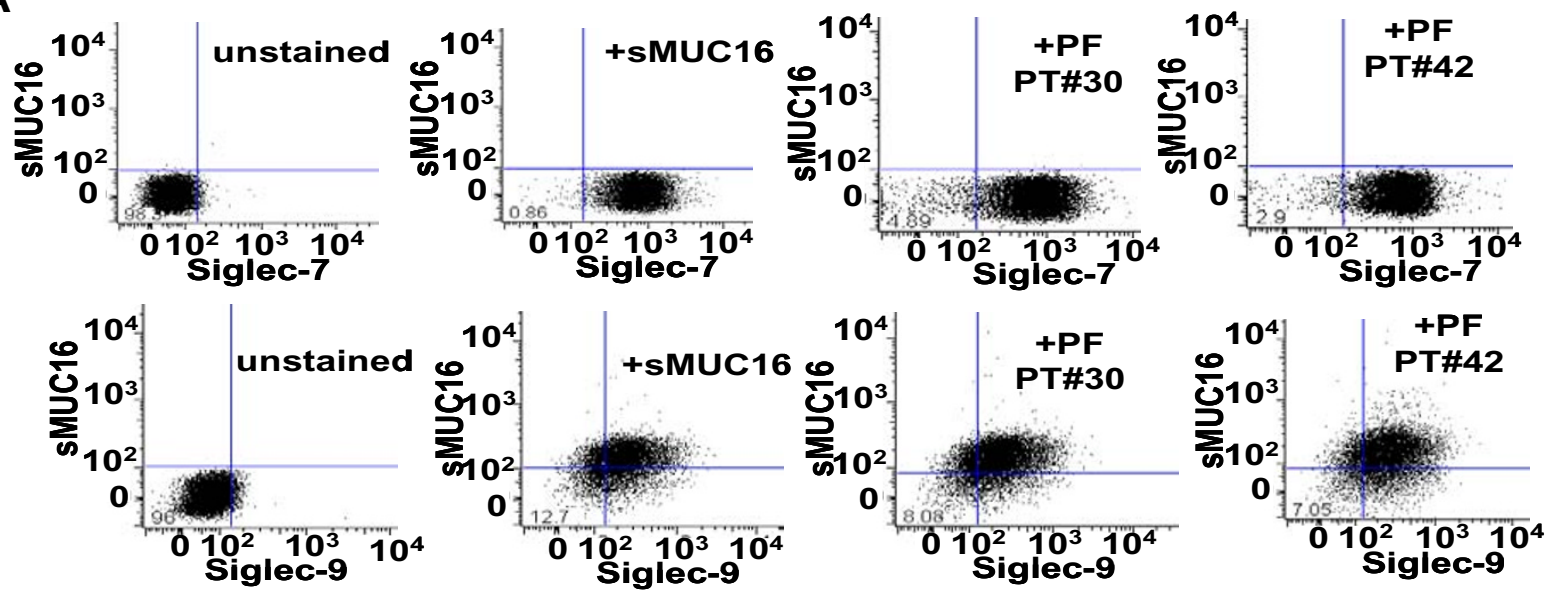

B

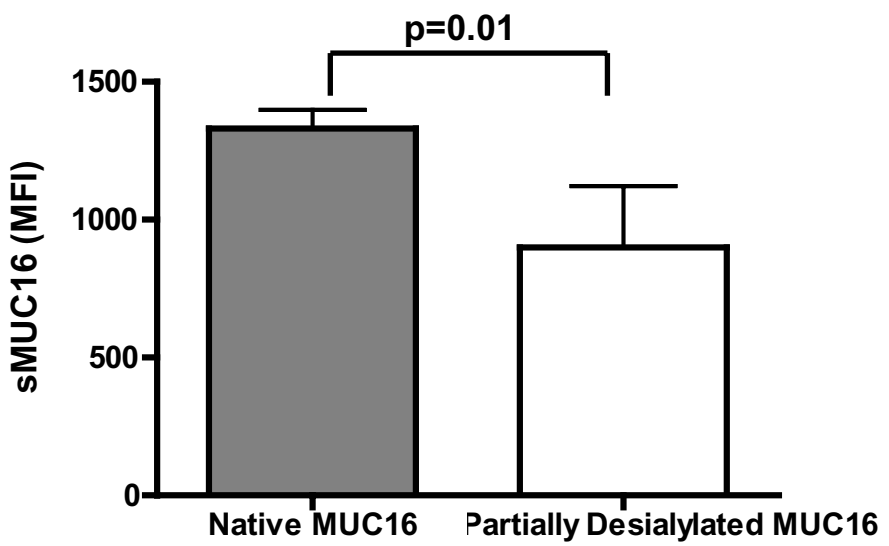

Figure 6 sMUC16-Siglec-9 binding specificity. $\boldsymbol{A}$, Siglec-7 (top panel) and Siglec-9 (bottom panel) expressing Jurkat cells were incubated for $24 \mathrm{~h}$ in media, media containing OVCAR-3 derived sMUC16 (50,000 U of CA125/ml), or in 10\% media containing 90\% peritoneal fluid (PF) from ovarian cancer patients (PT \#30 and PT \#42). The VK-8 antibody was used to detect binding of sMUC16 to the cells by flow cytometry. B, Siglec-9 expressing Jurkat cells were incubated with native and partially desialylated sMUC16 (50,000 U of CA125/ml) for $3 \mathrm{~h}$. Cells were labeled with VK8 and the amount of sMUC16 present on the surface was determined by flow cytometry. Mean and standard deviation of three separate experiments $(p<0.05)$ is shown.

tion of tyrosine residues in the ITIM and the recruitment and activation of the phosphatases SHP-1 and SHP-2 $[34,41]$. Because of the activation of these two phosphatases, binding of Siglec-9 to its ligands results in potent inhibition of NK cell and T cell anti-tumor functions $[34,41]$. Furthermore, engagement of Siglec-9 on monocytic cells results in the secretion of the immunosuppressive cytokine IL-10 [42].

In over 20 ovarian cancer patient samples analyzed to date we have invariably found sMUC16 attached to the surface of specific immune cell subsets [20]. Data presented in the current study demonstrates that sMUC16 predominantly binds to NK cells, B cells and monocytes via Siglec-9. These observations suggest that sMUC16Siglec-9 binding may trigger an inhibitory response in circulating cytotoxic immune cells even before they interact with the tumor. In other words, sMUC16-Siglec-9 binding may result in inhibitory priming of the immune cells leading to compromised anti-tumor immunity. The occurrence of immune suppression in ovarian cancer patients is a common observation [43-50]. sMUC16Siglec-9 binding is likely one of many molecular events that contribute to attenuation of anti-tumor immune responses. Our ongoing work is focused on determining the cytokine release and activation capacity of immune cells once sMUC16 binds to Siglec- 9 .

Competent Siglec-9pos immune cells entering the tumor microenvironment may adhere to the cancer cells via csMUC16. Siglecs have previously been shown to act as adhesion receptors. MUC1, another prominent member of the mucin family has been previously shown to be a ligand for Siglec-1 and Siglec-4a [36,51]. MUC1-Siglec-4a binding promotes the perineural invasion of pancreatic cancer cells [36]. Because Siglec-9 is an inhibitory receptor, the interaction between csMUC16 and Siglec-9 may not necessarily lead to enhanced immune recognition of 
A Wes Siglec-9 Jurkat + csMUC16 ${ }^{\text {pos OVC }}$

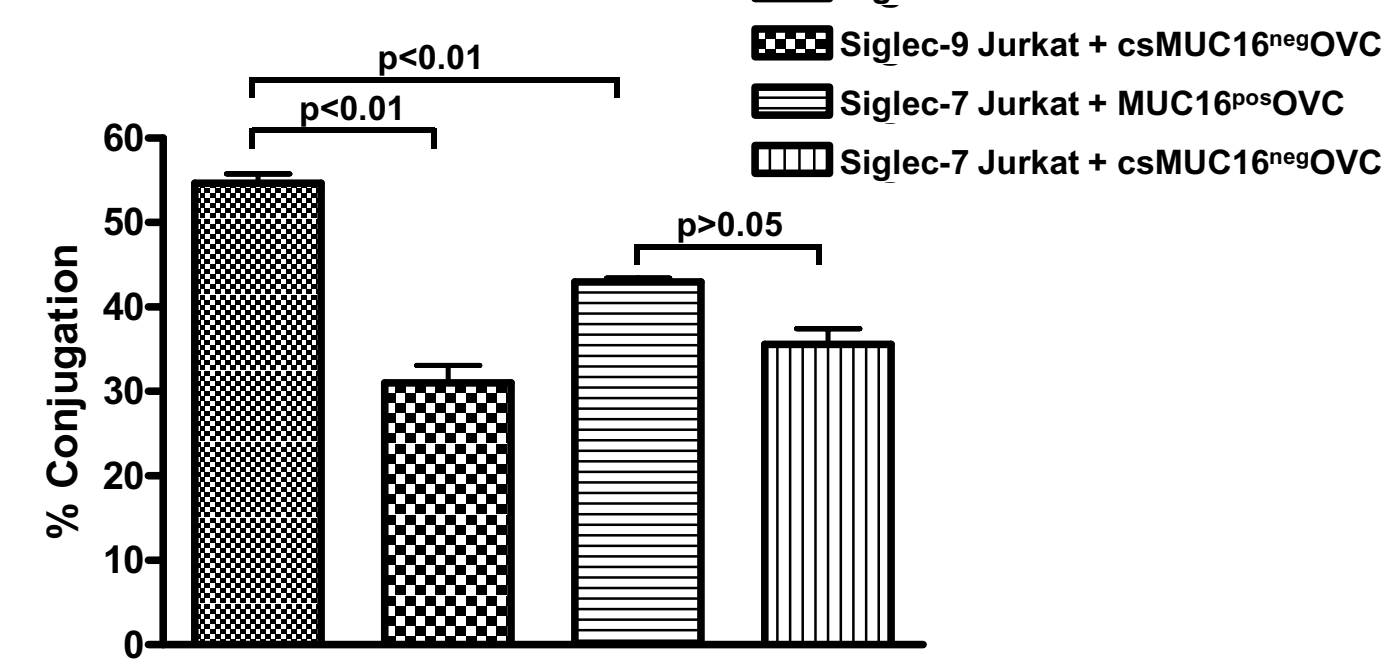

B
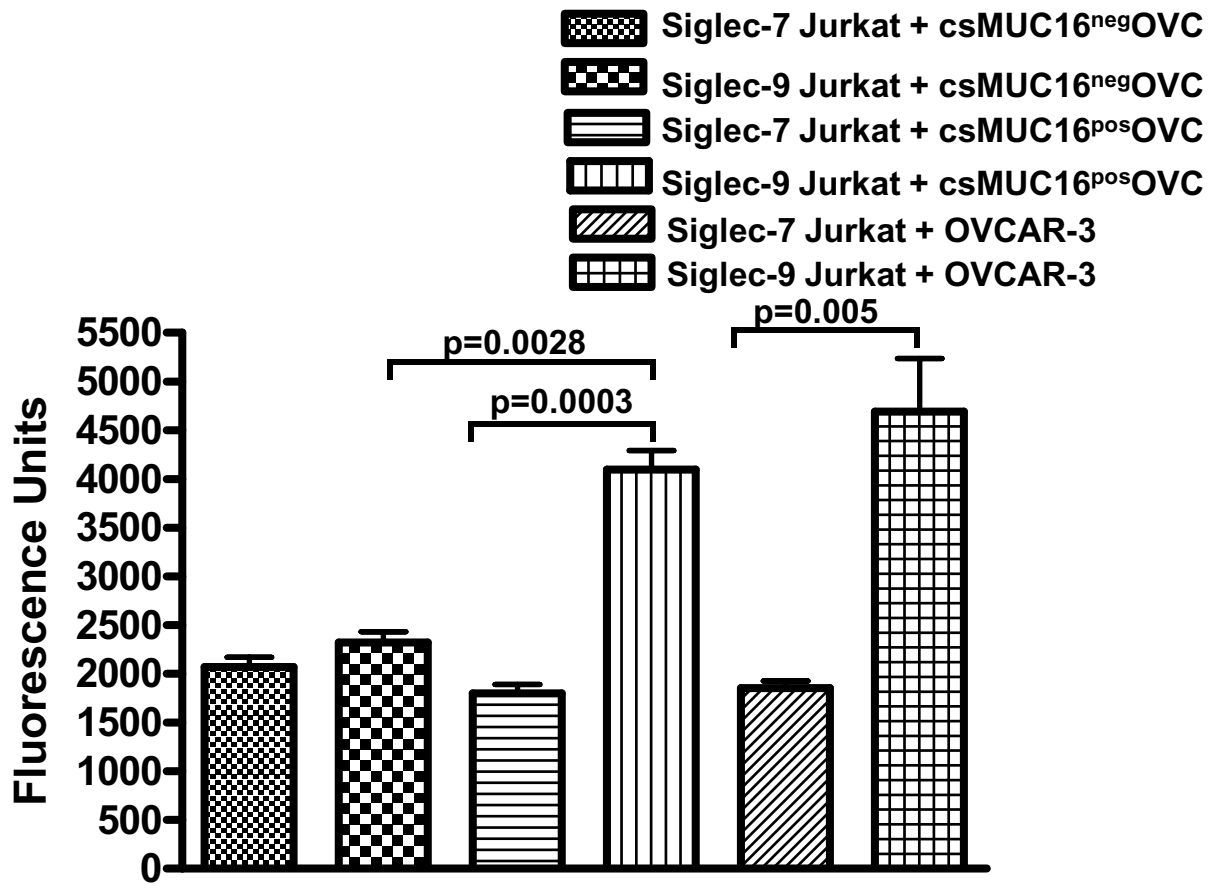

Figure 7 Siglec-9 binds to csMUC16 and mediates immune cell-target cell binding. $\boldsymbol{A}$, Siglec-9 expressing Jurkat cells were stained with CellTracker Blue and MUC16negOVC or MUC16posOVC cells were stained with CellTracker Green. The Jurkat cells and tumor targets were mixed in 1:1 ratio, incubated for $30 \mathrm{~min}$ at room temperature and conjugate formation was determined by flow cytometry as described previously [16]. Mean and standard deviation data for percent conjugation obtained from three independent flow cytometry experiments are summarized in the bar chart. $\boldsymbol{B}$, A direct 96-well plate-based cell adhesion assay was also used to measure binding of MUC16posOVC and Siglec-9 expressing Jurkat cells. Ovarian tumor cells were plated in 96-well plates. Calcein AM labeled Siglec-9pos or Siglec-7pos Jurkat cells were added to the wells. After 25 min incubation wells were washed three times and the amount of fluorescence remaining in the wells was measured. Data shown is mean of six experiments. 


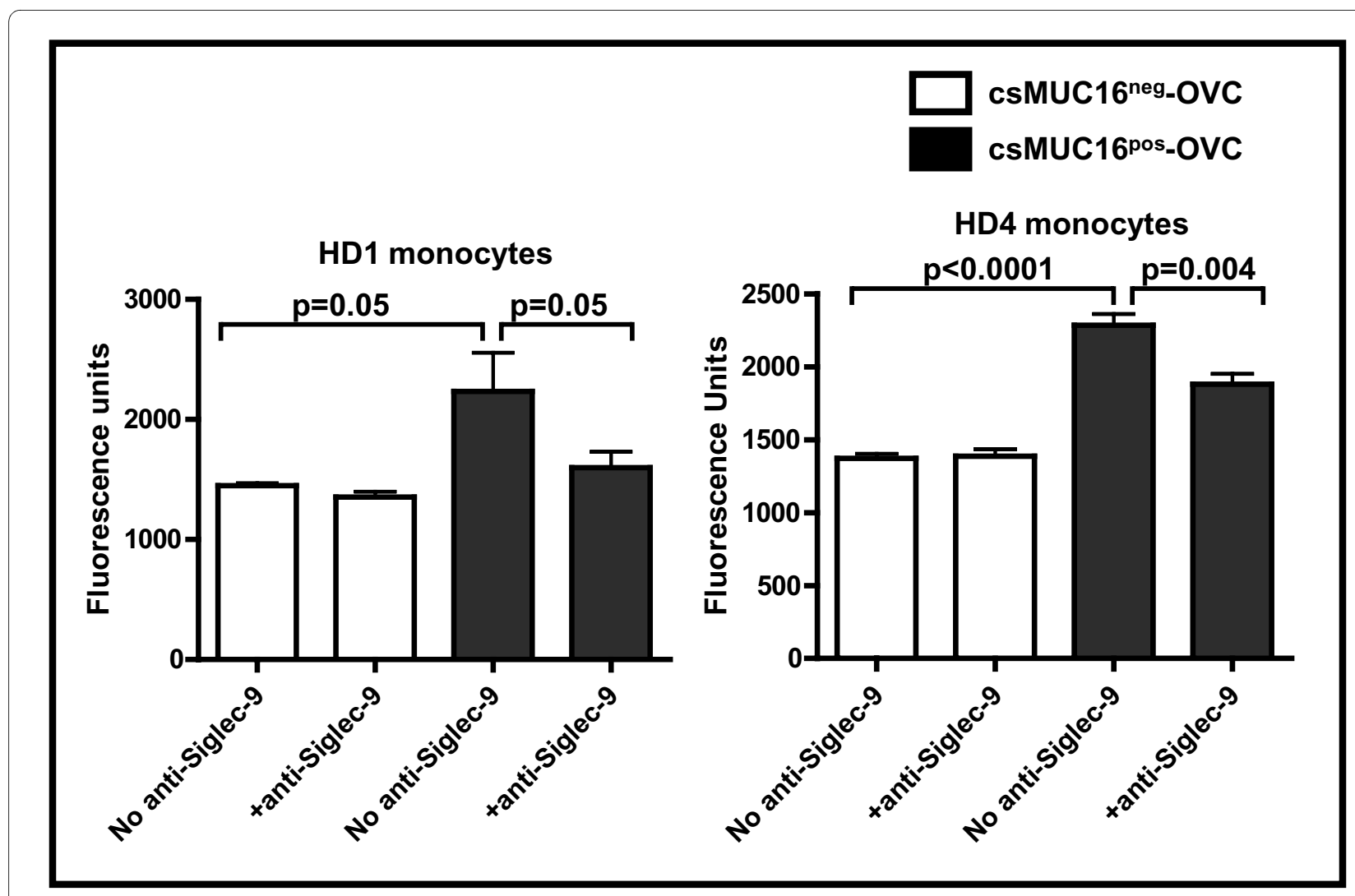

Figure 8 Monocytes bind to tumor cells via csMUC16-Siglec-9 interaction. Monocytes were purified from PBMC of healthy donors using the RosetteSep monocyte isolation kit (Stem Cell Technologies). The monocytes were labeled with Calcein AM and added to wells containing either CsMUC16 16 s-OVC or csMUC16 neg-OVC. After incubation and washing, the Calcein-labeled monocytes bound to the tumor cells were measured on a fluorescence plate reader. To demonstrate that binding was occurring via Siglec-9, matching Calcein AM-labeled monocytes were pre-treated with anti-Siglec-9 blocking antibody (Clone 191240, R\&D Systems) prior to conducting the cell binding assay. Data shown is mean of three independent measurements.

the tumor but instead cause protection of the cancer cells.

Finally, the anti-adhesive properties of csMUC16 allow the cancer cells to evade immune recognition by the Siglec-9neg immune cells. As shown in our other study [19], csMUC16 prevents efficient immune synapse formation between cancer cells and NK cells. Thus, the proadhesive and anti-adhesive properties of csMUC16 are of great benefit to the tumor and work in tandem to provide immune protection during the development and progression of ovarian cancer.

The identification of Siglec-9 as the receptor for MUC16 also has significance in the development of a novel test to monitor ovarian cancer progression. Serum CA125 levels are routinely monitored in patients who are already undergoing treatment for ovarian cancer. A decrease in serum CA125 indicates a positive response to the treatment regimen, whereas increase in this marker above a nadir concentration suggests recurrence of the tumor $[13,14,52]$. One major factor that impairs efficient detection of CA125 (MUC16) and other mucinous cancer antigens in the sera is their degradation by the liver. Circulating mucins are rapidly cleared by hepatic reticuloendothelial cells via lectins specific for galactose $/ \mathrm{N}$ acetylgalactosamine (tolerating $\alpha 2,3$-sialylation), mannose/ $\mathrm{N}$-acetylgalactosamine-4-sulfate, and hyaluronan receptors and the scavenger receptors [53]. Hepatic uptake of mucins therefore hampers efficient detection of these tumor antigens in cancer patients. The binding of sMUC16 to Siglec-9 on the surface of specific immune subsets may preclude the uptake and degradation of the mucin. Therefore, immune cell bound sMUC16 may prove to be a more reliable source for measuring the changes in CA125 levels in ovarian cancer patients. We are currently investigating the potential of monitoring immune cell bound sMUC16 as a more sensitive diagnostic assay to predict recurrence of ovarian cancer.

\section{Conclusions}

In this study we have identified Siglec-9 as the immune cell receptor for MUC16. Our previous studies have indicated that sMUC16 and csMUC16 serve an important 
immunomodulatory role, allowing tumor cells to escape recognition by NK cells. Siglec- 9 is an inhibitory receptor. The demonstration that sMUC16 and csMUC16 are ligands of Siglec-9 now allows us to further define the molecular mechanisms leading to the attenuation of immune responses by this mucin.

\section{Methods \\ Sample Processing}

The peripheral blood and peritoneal fluid samples were obtained from nine epithelial ovarian cancer patients (designated PT\#24, PT\#36, etc) recruited at the time of their initial diagnosis. All patients and healthy donors (designated HD\#1, HD\#2, etc) signed an informed consent, and the studies were approved by the Institutional Review Board of University of Wisconsin-Madison. The peripheral blood and peritoneal fluid samples were processed as described previously [20]. The serum CA125 levels of patients and healthy donors and other demographic information is provided as Supplementary Table 1.

\section{Flow Cytometry}

Cryopreserved or freshly obtained mononuclear cells from the peripheral blood or peritoneal fluid samples were analyzed. The cells were processed and labeled with antibodies as described previously [20]. For blocking purposes, cells were first incubated for 15 minutes with goat antibody before staining with the anti-MUC16 antibody VK8, or the anti-galectin-1 antibody, LGALS-1 (Abnova, Taipei, Taiwan). After washing, FITC- or APC-conjugated goat anti-mouse (GAM) secondary antibody was added at $1: 100$ or $1: 150$ dilutions, respectively and the cells were incubated with mouse IgG for 15 minutes to block any additional Fab sites on the GAM secondary. The cells were then incubated with various cocktails of directly conjugated antibodies to stain for CD3 (APCCy7), CD45 (PerCP-Cy5.5), CD33 (PerCP-Cy5.5), CD56 (Alexa 700), CD16 (PE-Cy7), CD19 (PE), Siglec-9 (FITC), or Siglec-7 (PE). After a final wash, cells were resuspended in $300 \mu \mathrm{L}$ of phosphate buffered saline containing $1 \%$ bovine serum albumin (PBS-BSA). Immediately before data acquisition on an LSRII (Beckton Dickinson) flow cytometer, the viability indicator DAPI (1:300; BD Biosciences) was added to each sample. Each fluorophore was compensated by automatic computerized compensation. To compare the values of samples analyzed on different days, SPHERO ${ }^{\mathrm{m}}$ Rainbow Fluorescent Particles (BD Biosciences) were used to set instrument voltages. Data was analyzed by FlowJo software (v. 8.5.3, TreeStar) and data plotting and statistical analysis was done using GraphPad Prism software (v. 4, GraphPad Software, Inc.).

\section{Western and Far-Western Blotting}

The sMUC16 (isolated from culture media of OVCAR-3 cells as described earlier [10]) and the bovine glycoprotein, fetuin, were separated on a $4 \%$ stacking/7.5\% resolving gel. The proteins were transferred to PVDF membrane, blocked with BSA and overlaid with either the VK-8 antibody or Siglec-9-human Fc chimera (2.5 nM) (R\&D Systems). Horseradish peroxidase conjugated goat anti-mouse or a goat anti-human Fc secondary antibodies were used for detection.

For inhibition of Siglec-9 binding to sMUC16 by fetuin or asialofetuin, the Siglec-9-human Fc was pre-incubated for 5 min with 3-fold molar excess of fetuin or asialofetuin. The Siglec-9-human-Fc along with the fetuin or asialofetuin was overlaid on membranes blotted with sMUC16 and binding was detected as described above.

\section{Desialylation of MUC16}

sMUC16 (2000 U of CA125) isolated from the conditioned media of OVCAR-3 cells was treated with $1 \mathrm{U}$ of neuraminidase from Clostridium perfringens (Sigma Aldrich) in phosphate buffered saline ( $\mathrm{pH} 7.4$ ) in a total of $100 \mu \mathrm{L}$ for $16 \mathrm{~h}$ at $37^{\circ} \mathrm{C}$. Control sMUC16 samples were treated under identical conditions except that neuraminidase was not added.

For desialylation experiments of immune cells, PBMC $\left(1-2 \times 10^{6}\right)$ freshly isolated from ovarian cancer patients were resuspended in $500 \mu \mathrm{l}$ phosphate buffered saline ( $\mathrm{pH} 7.4)$. Neuraminidase $(0.5 \mathrm{U} / \mathrm{ml})$ was added and the cells were incubated at $37^{\circ} \mathrm{C}$ for $15 \mathrm{~min}$. For controls, PBMC's from the same patients were incubated under identical conditions in buffer but neuraminidase was not added. After digestion, the test and control cells were washed with phosphate buffered saline containing $1 \%$ bovine serum albumin and sMUC16 bound to the cells was determined by flow cytometry.

\section{Binding of sMUC16 to Siglec expressing Jurkat cells}

The Siglec-7pos and Siglec-9pos Jurkat cells were developed as described in a previous report [34]. The cells were washed in culture media (RPMI 1640 containing 10\% fetal calf serum, non-essential amino acids and G418) and incubated for different time points in media containing native or desialylated sMUC16 (25,000-50,000 U CA125/ $\mathrm{ml}$ ) isolated from OVCAR-3 cells, or in a mixture of $90 \%$ peritoneal fluid, and $10 \%$ fetal calf serum. Following incubation, the cells were washed twice and stained for flow cytometry as described above.

\section{Cell conjugation assays}


ously designated as \#7 cells) and the corresponding 


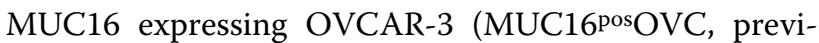
ously designated as \#12 cells) sublines were obtained as reported previously [16,37]. Siglec-9 and Siglec-7expressing Jurkat cells were labeled with $5 \mu \mathrm{M}$ CellTracker Blue (Invitrogen) for 25 minutes at $37^{\circ} \mathrm{C}$ in $5 \%$ $\mathrm{CO}_{2}$. Concurrently, MUC16 ${ }^{\text {negOVC }}$ and MUC16 ${ }^{\text {pos OVC }}$ were harvested and dyed with 1.25 pM CellTracker Green (Invitrogen) under identical conditions. After washing off excess dye with PBS, cells were resuspended in PBS containing 1\% BSA. Siglec-9 and Siglec-7 expressing Jurkat cells were placed in flow tubes with either

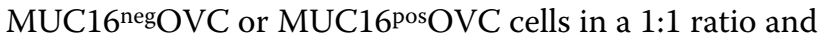
centrifuged for 2 minutes at $100 \mathrm{~g}$. Tubes were incubated for 30 minutes at room temperature, and vortexing was avoided. Immediately before data acquisition on an LSRII (Beckton Dickinson) flow cytometer, the viability indicator DAPI (BD Biosciences) was added to each sample.

For microtiter plate based assays, the MUC16 ${ }^{\text {pos_OVC, }}$ MUC16 ${ }^{\text {neg-OVC }}$ and OVCAR-3 cells $\left(1 \times 10^{5} /\right.$ well $)$ were cultured for two days in 96-well plates. On the day of the assay, Siglec-9 and Siglec-7 expressing Jurkat cells or monocytes isolated from the blood of healthy donors were labeled with Calcein AM $(0.1 \mu \mathrm{M})$ for 30 mins at $37^{\circ} \mathrm{C}$. Calcein AM labeled Jurkat cells or monocytes $(1 \times$ $10^{5} /$ well) were added to wells containing the ovarian tumor targets. Monocytes were freshly isolated using the RosetteSep monocyte isolation kits (Stem Cell Technologies). Tissue culture plates were incubated for $25 \mathrm{~min}$ at $37^{\circ} \mathrm{C}$ in a $5 \% \mathrm{CO}_{2}$ environment. After incubation, wells were washed 4-6 times with PBS containing 1\% BSA. The plates were analyzed on a fluorescent plate reader to determine the binding of Siglec-9 and Siglec-7 expressing Jurkat cells to the ovarian tumor targets.

\section{Additional material}

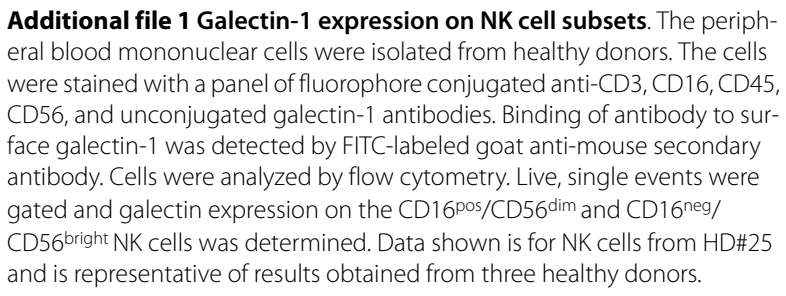

\section{Competing interests}

$J A B, J C$, and MSP are co-inventors on a United States patent application filed through the Wisconsin Alumni Research Foundation (WARF) on the use of immune cell bound SMUC16 as a diagnostic assay. As Co-inventors, JAB, JC, and MSP have received research funding and minor compensation from WARF. None of the other authors have any financial conflict of interest.

\section{Authors' contributions}

JAB conducted the majority of the research SH, JAAG, SP, and AK conducted some of the experiments and provided technical support. SA and HJG provided reagents for galectin-1 analysis and helped in designing galectin expression experiments. JPC provided the Siglec-9 and Siglec-7 reagents and provided intellectual input for this manuscript. JC provided technical and clinical expertise for this project. MSP is the corresponding author and helped in study design, data interpretation and preparation of the manuscript. All authors have read and approved the final version of this manuscript.

\section{Acknowledgements}

We thank Dr Beatrice Yin for providing us the anti-MUC16 antibody VK-8 and Dr. Alexander Rakhmilevich for critical reading of the manuscript. Funding for this research was provided by grants from the Department of Defense (\#W81XWH-04-1-0102), Ovarian Cancer Research Fund (UW/UWM.05), UW School of Medicine and Public Health Wisconsin Partnership Program, a charitable donation from Jean McKenzie, and start-up funds from the Department of Obstetrics and Gynecology to MSP, a T32 training grant (JAAG), research grants (GM60938 and Al50143) to JCP, and a grant of the research initiative LMU excellent to HJG. We are deeply grateful to Kathy Schell for her advice and help and acknowledge the support provided by the University of Wisconsin Comprehensive Cancer Centers Flow Cytometry facility which is supported by a core grant (CA14520) from the National Institutes of Health.

\section{Author Details}

'Department of Obstetrics and Gynecology, University of Wisconsin-Madison, Madison, WI; USA, 2Institute for Physiological Chemistry, Faculty of Veterinary Medicine, Ludwig-Maximilians-University, Munich, Germany, ${ }^{3}$ Department of Microbiology and Infectiology, Universite de Sherbrooke, Sherbrooke, Canada and 4 The Scripps Research Institute, Departments of Chemical Physiology and Molecular Biology, La Jolla, CA, USA

Received: 20 January 2010 Accepted: 24 May 2010

Published: 24 May 2010

References

1. Zeimet AG, Offner FA, Muller-Holzner E, Widschwendter M, Abendstein B, Fuith LC, Daxenbichler G, Marth C: Peritoneum and tissues of the female reproductive tract as physiological sources of CA-125. Tumour Biol 1998, 19:275-282.

2. Argueso P, Spurr-Michaud S, Russo CL, Tisdale A, Gipson IK: MUC16 mucin is expressed by the human ocular surface epithelia and carries the H185 carbohydrate epitope. Invest Ophthalmol Vis Sci 2003, 44:2487-2495.

3. Gipson IK, Blalock T, Tisdale A, Spurr-Michaud S, Allcorn S, Stavreus-Evers A, Gemzell K: MUC16 is Lost from the Uterodome (Pinopode) Surface of the Receptive Human Endometrium: In Vitro Evidence That MUC16 Is a Barrier to Trophoblast Adherence. Biol Reprod 2007, 78:134-142.

4. Davies JR, Kirkham S, Svitacheva N, Thornton DJ, Carlstedt I: MUC16 is produced in tracheal surface epithelium and submucosal glands and is present in secretions from normal human airway and cultured bronchial epithelial cells. Int J Biochem Cell Biol 2007, 39:1943-1954.

5. Yin BW, Dnistrian A, Lloyd KO: Ovarian cancer antigen CA125 is encoded by the MUC16 mucin gene. Int J Cancer 2002, 98:737-740.

6. Yin BW, Lloyd KO: Molecular cloning of the ca125 ovarian cancer antigen. identification as a new mucin, muc16. J Biol Chem 2001, 276:27371-27375

7. O'Brien TJ, Beard JB, Underwood $\sqcup$, Dennis RA, Santin AD, York L: The CA 125 gene: an extracellular superstructure dominated by repeat sequences. Tumour Biol 2001, 22:348-366.

8. Hattrup CL, Gendler SJ: Structure and Function of the Cell Surface (Tethered) Mucins. Annu Rev Physiol 2007, 70:431-457.

9. O'Brien TJ, Beard JB, Underwood LJ, Shigemasa K: The CA 125 gene: a newly discovered extension of the glycosylated $\mathrm{N}$ - terminal domain doubles the size of this extracellular superstructure. Tumour Biol 2002, 23:154-169

10. Kui Wong N, Easton RL, Panico M, Sutton-Smith M, Morrison JC, Lattanzio FA, Morris HR, Clark GF, Dell A, Patankar MS: Characterization of the oligosaccharides associated with the human ovarian tumor marker CA125. J Biol Chem 2003, 278:28619-28634.

11. Kabawat SE, Bast RC Jr, Bhan AK, Welch WR, Knapp RC, Colvin RB: Tissue distribution of a coelomic-epithelium-related antigen recognized by the monoclonal antibody OC125. Int J Gynecol Pathol 1983, 2:275-285.

12. Niloff JM, Klug TL, Schaetzl E, Zurawski VR Jr, Knapp RC, Bast RC Jr: Elevation of serum CA125 in carcinomas of the fallopian tube, 
endometrium, and endocervix. Am J Obstet Gynecol 1984, 148:1057-1058.

13. Buller RE, Berman ML, Bloss JD, Manetta A, DiSaia PJ: Serum CA125 regression in epithelial ovarian cancer: correlation with reassessment findings and survival. Gynecol Oncol 1992, 47:87-92.

14. Rustin GJ: Use of CA-125 to assess response to new agents in ovarian cancer trials. J Clin Oncol 2003, 21:187s-193s.

15. Rump A, Morikawa Y, Tanaka M, Minami S, Umesaki N, Takeuchi M, Miyajima A: Binding of ovarian cancer antigen CA125/MUC16 to mesothelin mediates cell adhesion. J Bio/ Chem 2004, 279:9190-9198.

16. Gubbels JA, Belisle J, Onda M, Rancourt C, Migneault M, Ho M, Bera TK, Connor J, Sathyanarayana BK, Lee B, Pastan I, Patankar MS: MesothelinMUC16 binding is a high affinity, $\mathrm{N}$-glycan dependent interaction that facilitates peritoneal metastasis of ovarian tumors. Mol Cancer 2006 5:50.

17. Bergan L, Gross JA, Nevin B, Urban N, Scholler N: Development and in vitro validation of anti-mesothelin biobodies that prevent CA125/ Mesothelin-dependent cell attachment. Cancer Lett 2007, 255:263-274.

18. Patankar MS, Yu J, Morrison JC, Belisle JA, Lattanzio FA, Deng Y, Wong NK, Morris HR, Dell A, Clark GF: Potent suppression of natural killer cell response mediated by the ovarian tumor marker CA125. Gynecol Oncol 2005, 99:704-713.

19. Gubbels JA, Felder M, Horibata S, Belisle JA, Kapur A, Holden H, Petrie S, Migneault M, Rancourt C, Connor JP, Patankar MS: MUC16 provides immune protection by inhibiting synapse formation between NK and ovarian tumor cells. Mol Cancer 2010, 9:11.

20. Belisle JA, Gubbels JA, Raphael CA, Migneault M, Rancourt C, Connor JP, Patankar MS: Peritoneal natural killer cells from epithelial ovarian cancer patients show an altered phenotype and bind to the tumour marker MUC16 (CA125). Immunology 2007, 122:418-429.

21. Lloyd $\mathrm{KO}$, Yin BW, Kudryashov V: Isolation and characterization of ovarian cancer antigen CA 125 using a new monoclonal antibody (VK8): identification as a mucin-type molecule. Int J Cancer 1997, 71:842-850.

22. Bast RC Jr, Feeney M, Lazarus H, Nadler LM, Colvin RB, Knapp RC: Reactivity of a monoclonal antibody with human ovarian carcinoma. J Clin Invest 1981, 68:1331-1337.

23. Bast RC Jr, Klug TL, St John E, Jenison E, Niloff JM, Lazarus H, Berkowitz RS, Leavitt T, Griffiths CT, Parker L, Zurawski VR Jr, Knapp RC: A radioimmunoassay using a monoclonal antibody to monitor the course of epithelial ovarian cancer. N Engl J Med 1983, 309:883-887.

24. Goodell CAR, Belisle JA, Gubbels JAA, Migneault M, Rancourt C, Connor JP, Kunnimalaiyaan M, Kravitz R, Tucker W, Zwick M, Patankar MS: Characterization of the Tumor Marker Muc16 (CA125) Expressed by Murine Ovarian Tumor Cell Lines and Identification of a Panel of CrossReactive Monoclonal Antibodies. Journal of Ovarian Research 2009, 2:8.

25. Nagler A, Lanier LL, Cwirla S, Phillips JH: Comparative studies of human FCRIII-positive and negative natural killer cells. J Immunol 1989, 143:3183-3191.

26. Cooper MA, Fehniger TA, Turner SC, Chen KS, Ghaheri BA, Ghayur T, Carson WE, Caligiuri MA: Human natural killer cells: a unique innate immunoregulatory role for the CD56(bright) subset. Blood 2001, 97:3146-3151.

27. Seelenmeyer C, Wegehingel S, Lechner J, Nickel W: The cancer antigen CA125 represents a novel counter receptor for galectin-1. J Cell SCi 2003, 116:1305-1318.

28. Hanna J, Bechtel P, Zhai Y, Youssef F, McLachlan K, Mandelboim O: Novel insights on human NK cells' immunological modalities revealed by gene expression profiling. J Immunol 2004, 173:6547-6563.

29. Koopman LA, Kopcow HD, Rybalov B, Boyson JE, Orange JS, Schatz F, Masch R, Lockwood CJ, Schachter AD, Park PJ, Strominger JL: Human decidual natural killer cells are a unique NK cell subset with immunomodulatory potential. J Exp Med 2003, 198:1201-1212.

30. Falco M, Biassoni R, Bottino C, Vitale M, Sivori S, Augugliaro R, Moretta L, Moretta A: Identification and molecular cloning of p75/AIRM1, a novel member of the sialoadhesin family that functions as an inhibitory receptor in human natural killer cells. J Exp Med 1999, 190:793-802.

31. Zhang JQ, Nicoll G, Jones C, Crocker PR: Siglec-9, a novel sialic acid binding member of the immunoglobulin superfamily expressed broadly on human blood leukocytes. J Biol Chem 2000, 275:22121-22126.
32. Angata $T$, Varki A: Cloning, characterization and phylogenetic analysis of Siglec-9, a new member of the CD33-related group of Siglecs. Evidence for co-evolution with sialic acid synthesis pathways. $J$ Biol Chem 2000, 275:22127-22135.

33. Varki A, Angata T: Siglecs--the major subfamily of I-type lectins. Glycobiology 2006, 16:1R-27R.

34. Ikehara Y, Ikehara SK, Paulson JC: Negative regulation of T cell receptor signaling by Siglec-7 (p70/AIRM) and Siglec-9. J Bio/ Chem 2004, 279:43117-43125.

35. Burton RM: The Action Of Neuraminidase From Clostridium Perfringens On Gangliosides. J Neurochem 1963, 10:503-512.

36. Swanson BJ, McDermott KM, Singh PK, Eggers JP, Crocker PR, Hollingsworth MA: MUC1 is a counter-receptor for myelin-associated glycoprotein (Siglec-4a) and their interaction contributes to adhesion in pancreatic cancer perineural invasion. Cancer Res 2007, 67:10222-10229.

37. Boivin M, Lane D, Piche A, Rancourt C: CA125 (MUC16) tumor antigen selectively modulates the sensitivity of ovarian cancer cells to genotoxic drug-induced apoptosis. Gynecol Oncol 2009, 115:407-413.

38. Carlin AF, Uchiyama S, Chang YC, Lewis AL, Nizet V, Varki A: Molecular mimicry of host sialylated glycans allows a bacterial pathogen to engage neutrophil Siglec- 9 and dampen the innate immune response. Blood 2009, 113:3333-3336.

39. Hollingsworth MA, Swanson BJ: Mucins in cancer: protection and control of the cell surface. Nat Rev Cancer 2004, 4:45-60.

40. Moniaux N, Andrianifahanana M, Brand RE, Batra SK: Multiple roles of mucins in pancreatic cancer, a lethal and challenging malignancy. $\mathrm{Br} J$ Cancer 2004, 91:1633-1638.

41. Avril T, Floyd H, Lopez F, Vivier E, Crocker PR: The membrane-proximal immunoreceptor tyrosine-based inhibitory motif is critical for the inhibitory signaling mediated by Siglecs-7 and -9, CD33-related Siglecs expressed on human monocytes and NK cells. J Immunol 2004, 173:6841-6849.

42. Ando M, Tu W, Nishijima K, lijima S: Siglec-9 enhances IL-10 production in macrophages via tyrosine-based motifs. Biochem Biophys Res Commun 2008, 369:878-883.

43. Lai $P$, Rabinowich H, Crowley-Nowick PA, Bell MC, Mantovani G, Whiteside TL: Alterations in expression and function of signal-transducing proteins in tumor-associated $\mathrm{T}$ and natural killer cells in patients with ovarian carcinoma. Clin Cancer Res 1996, 2:161-173.

44. Rabinowich $H$, Suminami $Y$, Reichert TE, Crowley-Nowick P, Bell M, Edwards R, Whiteside TL: Expression of cytokine genes or proteins and signaling molecules in lymphocytes associated with human ovarian carcinoma. Int J Cancer 1996, 68:276-284.

45. Melioli G, Ferrari I, Casartelli G, Ragni N: Lymphocytes isolated from the peritoneal fluid of women with advanced ovarian carcinoma differ significantly from autologous peripheral blood lymphocytes. Gynecol Oncol 1993, 48:301-307.

46. Mantovani A, Allavena P, Sessa C, Bolis G, Mangioni C: Natural killer activity of lymphoid cells isolated from human ascitic ovarian tumors. Int J Cancer 1980, 25:573-582.

47. Allavena P, Zanaboni F, Rossini S, Merendino A, Bonazzi C, Vassena L, Mangioni C, Mantovani A: Lymphokine-activated killer activity of tumor-associated and peripheral blood lymphocytes isolated from patients with ascites ovarian tumors. J Nat/ Cancer Inst 1986, 77:863-868.

48. Lotzova E, Savary CA, Freedman R, Bowen JM: Natural immunity against ovarian tumors. Comp Immunol Microbiol Infect Dis 1986, 9:269-275.

49. Lotzova E, Savary CA, Freedman RS, Bowen JM: Natural killer cell cytotoxic potential of patients with ovarian carcinoma and its modulation with virus-modified tumor cell extract. Cancer Immunol Immunother 1984, 17:124-129.

50. Lotzova E, Savary CA, Freedman RS, Edwards CL, Wharton JT: Recombinant IL-2-activated NK cells mediate LAK activity against ovarian cancer. Int J Cancer 1988, 42:225-231.

51. Nath D, Hartnell A, Happerfield L, Miles DW, Burchell J, TaylorPapadimitriou J, Crocker PR: Macrophage-tumour cell interactions: identification of MUC1 on breast cancer cells as a potential counterreceptor for the macrophage-restricted receptor, sialoadhesin. Immunology 1999, 98:213-219.

52. Buller RE, Berman ML, Bloss JD, Manetta A, DiSaia PJ: CA 125 regression: a model for epithelial ovarian cancer response. Am J Obstet Gynecol 1991, 165:360-367. 
53. Wahrenbrock MG, Varki A: Multiple hepatic receptors cooperate to eliminate secretory mucins aberrantly entering the bloodstream: are circulating cancer mucins the "tip of the iceberg"? Cancer Res 2006, 66:2433-2441

doi: $10.1186 / 1476-4598-9-118$

Cite this article as: Belisle et al., Identification of Siglec-9 as the receptor for MUC16 on human NK cells, B cells, and monocytes Molecular Cancer 2010, 9:118

Submit your next manuscript to BioMed Central and take full advantage of:

- Convenient online submission

- Thorough peer review

- No space constraints or color figure charges

- Immediate publication on acceptance

- Inclusion in PubMed, CAS, Scopus and Google Scholar

- Research which is freely available for redistribution

Submit your manuscript at www.biomedcentral.com/submit 